\title{
An expert review on current approaches for endotoxin detection in various biological products
}

\author{
Mohammed A. Elkhateeb ${ }^{a}$, Walid F. Elkhatib ${ }^{* b, c}$, Mohammad M. Aboulwafa ${ }^{c}$ \\ ${ }^{a}$ National Organization for Research and Control of Biologicals, Doki, Giza, Egypt \\ ${ }^{b}$ Department of Microbiology and Immunology, School of Pharmacy \& Pharmaceutical Industries, Badr University \\ in Cairo (BUC), Entertainment Area, Badr City, Cairo, Egypt \\ ${ }^{c}$ Microbiology and Immunology Department, Faculty of Pharmacy, Ain Shams University, Cairo 11566, Egypt
}

\begin{abstract}
End toxin is heat-stable lip polysaccharide (LPS) present in the outer membrane of the cell wall of Gramnegative bacteria. All parenteral preparations, as well as tissue implants, must be with no pyrogenic level of endotoxin or other related materials because of their associated health hazards and serious clinical effects. Accordingly, detection and limiting endotoxin in various pharmaceutical and biological products represent crucial issues. Rabbit pyrogen test (RPT) and Limulus Amebocyte Lysate (LAL) test are two methods used for endotoxin detection and quantification. Endotoxin detection is one of the most critical quality control tests required by the Food and Drug Administration (FDA) for all parenteral drugs in their final stage. Both in vitro LAL test and in vivo RPT can complement and reinforce each other but in certain cases, they are not interchangeable and they together provide a comprehensive picture of any potential contamination whether by endotoxin or any other pyrogenic matters.
\end{abstract}

Keywords: Endotoxin, biological products, detection, LAL test, RPT

*Correspondence | Walid F. Elkhatib; Microbiology and Immunology Department, Faculty of Pharmacy, Ain Shams University, Cairo 11566, Egypt. Email: walid-elkhatib@ pharma.asu.edu.eg; walid2005faisal@yahoo.com

Citation | Elkhateeb MA, Elkhatib WF, Aboulwafa M M, 2019. An expert review on current approaches for endotoxin detection in various biological products. Arch Pharm Sci ASU 3(2): 142-153

DOI: $10.21608 /$ APS.2019.13058.1000

Print ISSN: 2356-8380. Online ISSN: 2356-8399.

Received 04 February 2019. Accepted 12 April 2019.

Copyright: ${ }^{{ }^{2}} 2019$ Elkhateeb et al. This is an open-access article licensed under a Creative Commons Attribution 4.0 International License (CC BY 4.0), which permits unrestricted use, distribution, and reproduction in any medium, provided the original author(s) and source are credited. Published by: Ain Shams University, Faculty of Pharmacy

\section{INTRODUCTION}

The term Endotoxin was introduced in the late 19th century to define the specific component of Gram-negative bacteria, which in charge of pathophysiological phenomena related to Gramnegative infections. At the end of the twentieth century, the chemical, physical properties and biological structures of endotoxin were gradually revealed as the result of using advanced apparatuses and analytical techniques. Highly chemical stability Lipopolysaccharide molecules are released to the environment with the death of bacteria cells (Gram-negative type) (Fig. 1). Injured intestinal mucosa allows the entrance of Lipopolysaccharide molecules to circulated blood of a human, which can initiate endotoxemia, symptoms of endotoxemia direct affecting the function and structure of cells and organs, starting with raised body temperature, followed by changing metabolic functions, then modification of hemodynamics, finally may be leading to septic shock. 


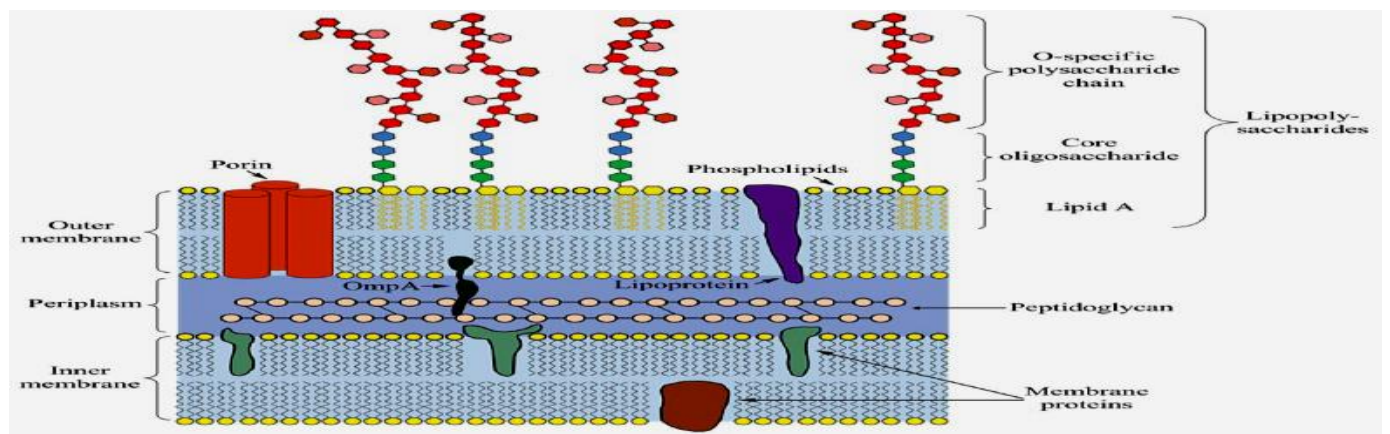

Fig. 1. Illustration of the lipopolysaccharide biochemical structure existing in the outer membrane of Gram-negative bacteria [17].

When LPS molecules enter the human blood circulating system via liver, various types of inflammatory cytokines $[\mathbf{1}, \mathbf{2}]$ as TNF, IL-6, platelet-activating factor, and others, are overexpressed by the innate immune system activation and this leading to systemic inflammatory response syndrome (SIRS), which has been reported as the main cause of death related to severe acute respiratory syndromes (SARS) [3, 4]. The high death rate related to endotoxin-induced shock still a critical clinical issue, especially in patients suffering from immunosuppressant and debilitated.

On the other hand, endotoxin is remaining and not removed when the microorganism is killed by the valid sterilization procedure; while that, the release of Lipopolysaccharide done upon the cell's death. Although the biological products after sterilized, the LPS of the organism still present if the product contaminated with Gramnegative organisms before sterilization. Detecting of endotoxin in the finished products is a very critical issue to ensure the safety of the sterilized biological products, medical devices, injectable drugs, water, and food quality.

The RPT has been used for more than 6 decades and has proven reliable for a great variety of products. This test was the first method approved by the FDA for Lipopolysaccharide testing. RPT was approved in the 1920s on the basis of the increase of rabbit temperature after injection of intravenous test solution Due to substantial similarities in sensitivity and specificity between the rabbit and the human's immune response, this test can be considered safe as a true pyrogen test, the RPT became the golden standard of pyrogen testing and has established a high level of security in pharmaceutical products. The sensitivity of man and rabbit for pyrogens is comparable. After intravenous injection of the test substance into a group of three rabbits, the individual changes in body temperatures are recorded over a defined period where it can detect endotoxin and nonendotoxin pyrogens (NEP) and therefore possesses a broad spectrum of applications. A negative test in a RPT is more significant than a positive one because the rabbit has a labile thermoregulatory mechanism (sometimes gives false-positive results). On the other hand, the dog has a much more stable thermoregulatory mechanism but is less sensitive to pyrogen than is the rabbit.

40th anniversary of LAL licensing by the US FDA, as an alternative to the US Pharmacopoeia's (USP) Pyrogen Test (PT) for endotoxin in human and veterinary drugs, is considered as a landmark for the pyrogen detection. Since this approval, LAL has become accepted worldwide to ensure the pyrogen safety of the world's pharmaceutical drug supply. The test components not only recognize bacteria (those of the Gram-negative type) but also fungi (those containing $\beta$-D-1, 3-glucan). Besides its inclusion in the USP as the Bacterial Endotoxin 
Test (BET), the LAL test is also included in the European Pharmacopoeia (European Pharmacopoeia Commission 1987) and the Japanese Pharmacopoeia (The Pharmacopoeia of Japan 1992). Although the RPT remains an official test, in most cases the BET has completely replaced the RPT.

While LAL has been criticized as not being a true pyrogen test, i.e., there are other pyrogens (fever-causing agents), endotoxin is the most common and most potent pyrogen and the most likely one (but not only one) pyrogen to contaminate injectable drugs and devices. The most important difference between the RPT and the LAL test is the failure of the latter to detect non-endotoxin pyrogens $[\mathbf{5}, \mathbf{6}]$.

\section{Special important of LPS (endotoxin) control}

The control of endotoxin contamination in parenteral manufacturing becomes very important because it's unique in nature, potent toxicity, high stability and ability to remain its toxicity after subjected to drastic conditions. The concern for endotoxin from a parenteral manufacturing contamination control perspective has overtaken concerns for guarding against "all pyrogens" that predominated the second half of almost a century of parenteral manufacturing. The paradigm shift of concern from "pyrogens" in general to "endotoxin" specifically started with the control of water use in the pharmaceutical industry and in-process control of pharmaceutical materials. United States Pharmacopeia (USP) [5] mention that " depending on availability the LAL test become suitable to alternative the pyrogen test for most final products".

\section{The ubiquity of LPS (Endotoxin)}

Endotoxin is "asymmetrical" high-molecularweight complexes present in the double layer of the outer membrane of the gram-negative organism, with critical protection function for
Gram-negative organism from its environment. In these organisms, the double layers were padded with a thin layer of peptidoglycan (PG). The unique properties of LPS structure have several tied correlative with its power to formulate defense mechanisms of the host cell. An individual cell from bacteria has been considered to include nearly 3.6 million lipopolysaccharide molecules present at space about $5 \mathrm{~mm}^{2}$ of a rated $6.7 \mathrm{~mm}^{2}$ from the whole surface area [7]. Thus, the outer membrane holds thereof four-quarters lipopolysaccharide and single-quarter from protein $[\mathbf{8 , 9}]$.

LPS particles are fateful for the survival of Gram-negative bacteria by providing immunologic, physiological, nutritional, pathological, transport functions, and structural integrity of bacteria. Thus, Gram-negative organisms completely lacking LPS are very rare to be found in nature [10].

The occurrence of Gram-negative organisms in almost every environment on the earth makes LPS one of the most prevalent complex organic molecules occurring in nature. Gram-negative bacteria have been isolated from diverse environments and localities including fresh and saltwater, soil, hot springs, and frigid oceans [11].

The molecules of LPS are released from bacteria cells upon lysis, dividing, consistent sloughing off of endotoxin in a way practically equivalent to the body shedding little bits of hair or skin and doom of bacterial cell. Assembling of solution rich in skeletons of dead bacteria of Gram-negative cellular remains and reach mammalian blood, they able to activate mammalian immune defense mechanisms in a very small amount (nanogram / kg body weight) [12].

\section{Stability of Endotoxin}

It is reported that endotoxin maintains the 
physical nature of the cell wall for the Gramnegative bacteria which assures strength enough to resist to 3 atm of turgor pressure, rigid enough to withstand upon exposure to extreme temperatures and $\mathrm{pH}$, and flexible enough to be able to expand several times their normal surface area [13]. Endotoxin is responsible for the physicochemical properties of the cell walls for Gram-negative bacteria. It has extreme heat stability and maintains its pyrogenicity after both ordinary steam, and normal dry heat sterilization. It easily penetrates filters purposed to whole bacterial removal from parenteral preparations. Only at dry heat sterilization using temperature exceeding $200{ }^{\circ} \mathrm{C}$ for more than 1 hour do they alleviate [14].

The LPS has an amphiphilic nature that serves as a flexible structure in solutions with hydrophobic lipid structure that allow adherence with hydrophobic surfaces as charcoal, glass, and plastic. The generality singular characters of lipopolysaccharide have participated with lipid bilayers, which give the common composition for all structures of the cell membranes [14].

LPS in hydrous unprompted double layers structures composed from hydrophobic "lipid A" ends with "fatty acid" tails are unobserved in the inner of the supra-molecular aggregate while the polysaccharide hydrophilic ends are faced to and subject to solubilization in the aqueous environment. Those increase the stability of lipopolysaccharide as a lipid double-layer tendency to liberate upon disrupted, thus aid to maintain the integrity of the structure's defense against the external climate $[\mathbf{1 5}, \mathbf{1 6}]$.

\section{Relative Pyrogenicity of endotoxin}

LPS (bacterial endotoxin) can be categorized, between others, as a substantial "pyrogen". Pyrogens are substances that, when injected intravenously cause rising in body temperature and will cause several pathophysiological effects.
The pyrogenic materials even reach blood circulation cause fever, chills, pupillary dilatation, decrease in respiration, nausea, malaise, increased arterial blood pressure and vasoconstriction of cutaneous tissues that develop extremely rapid to severe shock, often followed by death before the cause is even diagnosed [17, 18].

LPS makes a powerful influence in the host, which formulates very harmful effects rather than any other pyrogens, as is shown in the proportional small quantity of LPS required to stimulate the response of an immune system which is in the nanogram $/ \mathrm{kg}$. LPS is counted as one of the important hazard indications for a host in identification for microbial contamination [19, 20]. A very small amount of LPS creates an exaggerated host response. More efforts are expended to treat the complicated immune host response against endotoxin in the body. The complicated status appears from releasing of different mediators with different clinical effects of various inflammatory and proinflammatory mediators in addition to the synergistic effect of each other. Prostaglandin (PG) is the most match endogenous pyrogenic mediator to endotoxin $[\mathbf{5}$, 21, 22].

In the early $20^{\text {th }}$ century, when the pyrogen assay started no effort was spend to make quantitative detection for the minimum amount of endotoxin needed to produce rabbit's pyrogenic response. The pyrogenic dose-response curve in man is much steeper than it is in rabbits, although the minimum pyrogenic dose on a weight basis is about the same. Human is the most sensitive type of mammalians to endotoxin. A rabbit requires 10 times as much S. Typhi endotoxin as in man to produce the same degree of proportional febrile response [23]. Because of the hazard of endotoxin in humans, the effects of a higher dose of endotoxin cannot be studied. The exaggerating of human response to endotoxin 
with certain diseases as hepatic cirrhosis, and typhoid fever has been observed. On the other hand, also some diseases as malaria can decrease the host's toxic responsiveness to endotoxin [10, 24-26].

\section{Methods of endotoxin detection}

\subsection{Limulus Amoebocyte Lysate (LAL) test}

Limulus Amoebocyte Lysate existing in the blood of horseshoe crabs (Fig. 2), the LAL test technique depends on clotting reaction aid to quantitative detection of LPS based on clotting of the lysate reagent in contact with endotoxins. labeled "lysate sensitivity (ë)" is the conc. of ET

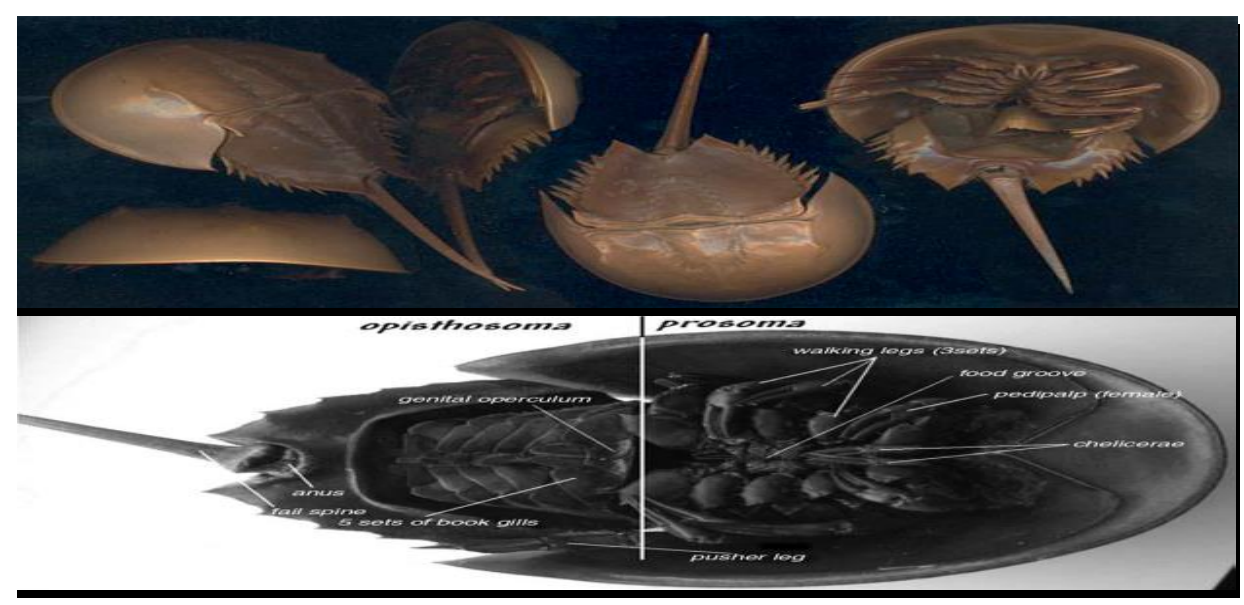

Fig. 2. horseshoe crabs and its body parts [17]

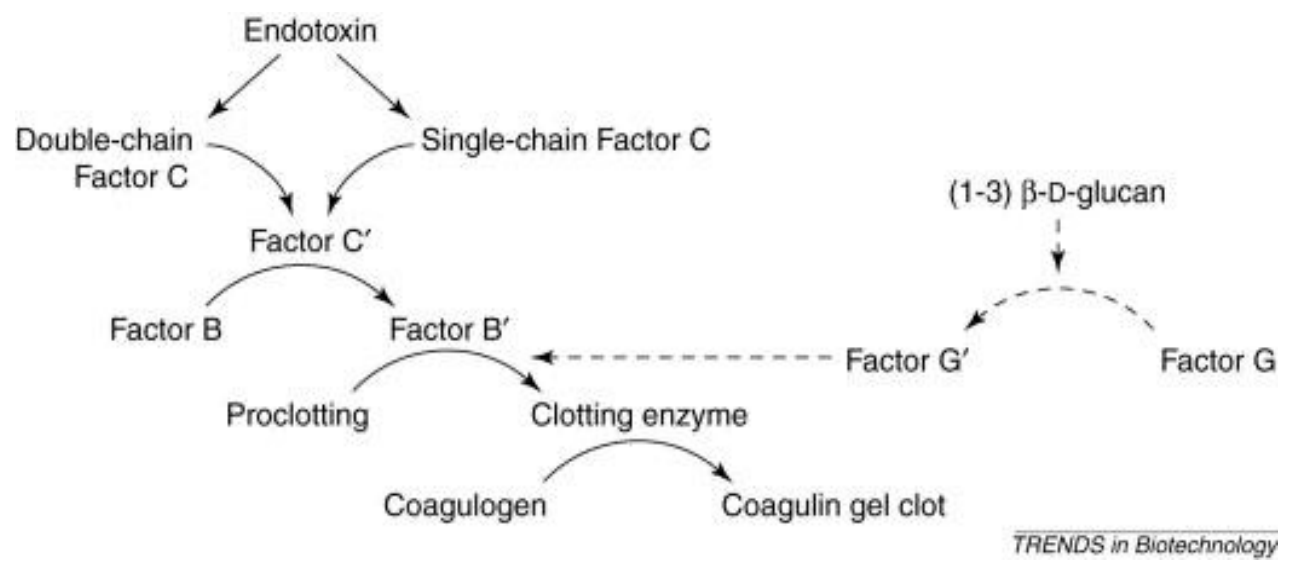

Fig. 3. Cascade of LAL test enzymatic reactions [30].

When bacteria come in contact with crab's blood, it cannot spread through the biochemical required to make the lysate to clot under optimum test conditions [27, 28].

The cascade of protease employed in the LAL test is started by the reaction between lipopolysaccharide and zymogen Factor C. then the activated Factor $\mathrm{C}$ stimulates the activation of Factor B, which able to converts the pro-clotting enzyme to the clotting enzyme. At the end of the cascade reaction, coagulin gel was formed through the 2 peptide bonds in coagulated are catalytically cleaved [29] (Fig. 3). 
system of the horseshoe crab due to the blood clotting strategy. The horseshoe crab's amoebocytes detect the endotoxin of Gramnegative bacteria, initiating a cascade of enzymatic reactions ending with the formation of a coagulin gel through the conversion of amoebocyte coagulogen to clottable protein. Fungi are not away from this sensitive defense mechanism. The horseshoe crab's amoebocytes respond in a similar way to fungal infection by induction of the clotting sequence through coagulin formation as a result of $\beta$-glucans which activate the protease enzyme factor $\mathrm{G}$ (the factor $\mathrm{C}$ in case of endotoxin). The horseshoe crab's amoebocytes are more sensitive to endotoxin by 1000 times than glucan. Different glucan molecular weights are required to activate factor $\mathrm{G}$ (from 3 to $100 \mathrm{kDa}$ ) [31] [32]. Doubling dilutions series is used to quantify endotoxin concentration in an object against LAL reagent under controlled temperature. Validation for each technician and each product is required to establish the LAL test as an accepted method. Internal standardization is required for valid assays $[\mathbf{1 7}, \mathbf{3 3}]$.

LAL gel clot technique is the simplest of LAL techniques where LAL reagent forms a firm gel with a specified endotoxin amount. The critical endotoxin amounts which clot the LAL reagent are known, so the formation of a clot is a function of the endotoxin sensitivity of the given LAL reagent, and testing dilution of a sample containing endotoxin. The concentration of endotoxin is calculated using sample dilution yielding no reaction (no clot formation/negative) with the less sensitive LAL reagent (higher critical Endotoxin content) unless exceeding the maximum valid dilution in several hours' process. Updated advanced techniques are used to develop the usual LAL test, resulting in faster and cheaper LPS detection techniques for quantity or quality such as the endpoint chromogenic LAL assay, kinetic LAL assay (turbidimetric and chromogenic) [34-36]. The principle of kinetic methods (turbidimetric and chromogenic), is the exact detection of alteration in color, and turbidity, respectively by light-scattering devices at endotoxin concentrations lower than those for gels. This kinetic methods can detect up to 0.001 EU/mL (Berzofsky 2007) [37, 38].

\subsubsection{Applications of LAL test}

Applications of the LAL test include (1) Diagnosis of acute UTI and CNS meningococcal infection. In these situations, the detection of endotoxin usually indicates the presence of living bacteria, (2) Estimate spoilage in different types of food (fish, milk, ground beef), air, determination of water quality, and for experimental determination of the ability of new anti-toxic-drugs to equilibrate the toxic effects of endotoxin, (3) Detection of Gram-negative bacterial endotoxin in injectable, surgical and medical devices, renal dialysis fluids and biological products in raw materials, in-process solutions, and finished product to ensure safety of these products to human use, (4) Detection of (1$3)-\beta$-D-glucan, which present in fungal cell walls, (5) Checking the clarity of pharmaceutical water (main ingredient and processing agent for all pharmaceutical and biological drugs and devices.) from endotoxin contamination $[27,39$. 41].

\subsubsection{LAL reagent water}

This water type was sterile and pyrogen-free and used in test procedure as endotoxin-free tested material as negative control and for dilution and reconstitution purposes $[39,40]$.

\subsubsection{Endotoxin standard solution}

Standard endotoxin was supplied in vials, each vial contains $500 \mathrm{ng}$ of purified lipopolysaccharide, freeze-dried in stabilized matrix prepared from E. coli strain 055:B5. 
According to manufacturer instructions, the content of each vial was reconstituted by LAL reagent water to the required concentration of the stock solution, when needed the stock solution was diluted to the required working concentration with LAL reagent water $[39,40]$.

\subsubsection{LAL Reagent}

This reagent (Limulus Amebocyte Lysate). It's supplied as a lyophilized powder, the reagent was supplied in different sensitivity $(0.25,0.125$ and $0.06 \mathrm{EU} / \mathrm{mL})[39,40]$.

\subsubsection{The validity of the LAL test by gel-clot technique}

\subsubsection{Validation of sample testing dilution}

For the LAL test to be valid, the sample should be tested at dilution not more than Maximum Valid Dilution (MVD). MVD is the maximum allowable dilution of a sample at which the endotoxin limit can be determined. The MVD can be calculated by using the following formula:

MVD $=$ endotoxin limit $x$ concentration of test solution

$\lambda$

Endotoxin limit can be obtained from pharmacopeia or calculated as follows: $\mathrm{K} / \mathrm{M}$

$\mathrm{K}$ is a threshold pyrogenic dose of endotoxin per kilogram of body mass, this dose is equal to 5 $\mathrm{EU} / \mathrm{kg}$ in case of intravenous injectable preparations and $0.2 \mathrm{EU} / \mathrm{kg}$ body mass in case of intrathecal injectable preparations.

$\mathrm{M}$ is the maximum recommended bolus dose of product per kilogram of body mass

\subsubsection{Confirmation of the labeled lysate sensitivity $(\lambda)$}

Labeled sensitivity $\lambda$ was confirmed by using 4 replicates expressed in $\mathrm{EU} / \mathrm{mL}$ of the lysate reagent before to use in the test. This confirmation was carried out for each lysate batch before being used in the test and this is carried out when the investigators use the batch of lysate for the first time.

\subsection{Test for absence of product interference}

The test preparation should not interfere with the test sample either by inhibiting or enhance the reaction. The test for interfering factors is repeated when any experimental conditions changes that influence the result of the test.

After determination of MVD, a serial dilution of the sample was prepared in a range not exceeding MVD, and tested for the absence of interference Four test reaction solutions (A, B, C and D) were prepared as the follows:

- solution A: (product dilution) and solution B (positive product control) using a dilution not greater than the MVD

- Solutions B and C (positive controls) contain the standard endotoxin at a concentration corresponding to twice the labeled lysate sensitivity

- Solution D (negative control) consists of water for LAL.

\subsection{Rabbit pyrogen test (RPT)}

Pyrogen test measuring the increase of temperature in rabbits after intravenous injection of sterile tested solution $[\mathbf{4 2}, \mathbf{4 3}]$.

\subsubsection{Validation of RPT}

For every RPT validity criteria should be checked and achieved, these validity criteria include:

A temperature variation between two successive readings during the initial temperature detection should be less than $0.2^{\circ} \mathrm{C}$.

$\checkmark \quad$ No rabbits had initial temperatures that differ from one another not exceed $1{ }^{\circ} \mathrm{C}$.

$\checkmark \quad$ No rabbits had an initial temperature 
higher than $39.8{ }^{\circ} \mathrm{C}$ or less than $38.0{ }^{\circ} \mathrm{C}$.

Rabbits used in an RPT where the mean rise in the rabbit's temperature exceeded $1.2{ }^{\circ} \mathrm{C}$ were permanently excluded [43].

\subsubsection{Rabbits manipulation and conditioning}

The rabbits used were healthy, adult and untreated and not less than $1.5 \mathrm{~kg}$ body weight. They have received a complete balanced diet free from antibiotics and kept individually in uniform, quiet, appropriate housing and environment. Animal feeding was withheld night prior test and until the test was completed.

\subsubsection{Testing conditions for preliminary and main tests}

The test was carried out in a quiet disturbance-free room to avoid the risk of exciting and temperature within the room was between $3{ }^{\circ} \mathrm{C}$ of that of the rabbit's living quarters before the test. Water was withheld during the test and the animals are placed into the retaining boxes where they were retained only by the loosely fitting neck - stocks and the rest of the body remain relatively free. The rabbits were put in the normal position for not less than 1 hour before the first record of temperature and remain in the boxes throughout the test. The temperature-sensing probe inserted into the rectum of the test rabbit to a depth of about $5 \mathrm{~cm}$ and should be kept there throughout the test period, the temperature of rabbits record $90 \mathrm{~min}$. before injection within $30 \mathrm{~min}$ interval. Then the initial temperature was calculated and rabbits whose temperature ranging from $38.0{ }^{\circ} \mathrm{C}$ to 39.8 ${ }^{\circ} \mathrm{C}$ were chosen for injection. The rabbit showing an increase in body temperature of more than 0.6 ${ }^{\circ} \mathrm{C}$ were excluded from the main test.

\subsubsection{Injection procedures}

Hair was removed from the place of injection at the rabbit ear and skin covering the marginal vain was disinfected by $70 \%$ ethanol. Besides the disinfection, the use of ethanol causes dilution of vain and easies the injection. The samples to be injected were pre-equilibrated at room temperature and injected over a period not more than four minutes for each rabbit unless otherwise prescribed in the pharmacopeial monograph. After injection, the rabbit's ear was wrapped with cotton to prevent bleeding. The temperatures of injected rabbits are recorded at $30 \mathrm{~min}$ interval over a test period of $180 \mathrm{~min}$.

\subsubsection{Preliminary test}

A preliminary test was performed 1-3 days before the main test for rabbits which were not used in pyrogen tests during the previous 2 weeks or newly received from the supplier or after spending 3 weeks from the last test in which the tested product does not pass pyrogen test (pyrogenic substance).

In this test, the rabbits were injected with sterile pyrogen-free $0.9 \%$ saline at a dose of $10 \mathrm{~mL} \mathrm{~kg}$.

\subsubsection{Main test}

In the main test three rabbits were used for each product from those shown the following characteristics: (1) Pass the preliminary test, (2) Reused only after 3 days from the last negative, (3) Pyrogen test or 3 weeks from last positive pyrogen test.

\subsubsection{Limitations of RPT}

The rabbit pyrogen test which is a qualitative biological test used for detection of pyrogens in parenteral preparations has several limitations that may include: (1) The rabbit's sensitivity to endotoxin contaminated preparations depends upon the strain of rabbit used and other different parameters as gender, age, and habitation circumstance. But the pyrogen detection limit is restricted due to it depends on the maximum allowed volume which is not more than $10 \mathrm{ml} / \mathrm{kg}$ body weight, thus the pyrogen detection limit does not exceed 50-350 picograms (i.e., 0.5-3.5 
EU) of lipopolysaccharide per $\mathrm{Kg}$, (2) The RPT is a qualitative, not a quantitative test (the result interpreted as pass or fail only) so, the RPT is not convenient for the determination of pyrogen level. RPT cannot be fully standardized, (3) The febrile response and sensitivity to endotoxin in RPT is rabbit age-dependant. The fever response for the rabbits to some materials such as biologicals for human use may be different from that on humans due to species differences. That is after performing RPT the temperature of rabbits may increase and give a positive result which indicates pyrogens contamination according to the GMP requirements and mentioned monographs. However, human use of this product may not cause the same response and vice versa. So, the permanent gap between the observed, and expected fever response in rabbits comparing to humans is fundamental, (4) The rabbit pyrogen test cannot detect endotoxin in LAL reagent water (water for reconstitution) which may contain a very low EL. Radiopharmaceutical products cannot be tested by the rabbit pyrogen tests $[44, \mathbf{4 5}]$.

\section{Conclusion}

Finally, from this review, we can conclude that the LAL test and RPT can complement and reinforce each other but in certain cases, they are not interchangeable and they together provide a comprehensive picture of any potential contamination whether by endotoxin or any other pyrogenic matters. Both methods (LAL test and RPT) used for endotoxin detection can be applied for various biological products including therapeutic proteins, vaccines, and other biological products. However, the LAL test is more accurate, fast, economical, and sensitive than RPT, however, the LAL test has limitations in some tested products, such as reaction suppression and interference between the reagent and product ingredients and with no value in the detection of non-endotoxin pyrogenic materials.

\section{Declarations}

\section{Ethics approval and consent to participate}

Not applicable

\section{Consent to publish}

Not applicable

\section{Availability of data and materials}

All data generated or analyzed during this study are included in this published article in the main manuscript.

\section{Competing interests}

The authors declare that no competing interests exist

\section{Funding Statement}

No funding source was received.

\section{Authors' contributions}

All authors have read, revised and approved the final manuscript.

\section{Acknowledgment}

The authors would like to acknowledge all colleagues in the Microbiology and Immunology Department, Faculty of Pharmacy, Ain Shams University and National Organization for Research and Control of Biologicals for their support and encouragement.

\section{REFERENCES}

1. Bhattacharyya J., S. Biswas, and A. G. Datta, Mode of action of endotoxin: role of free radicals and antioxidants. Current medicinal chemistry, 2004. 11(3): p. 359-368.

2. Stewart, Rachel K Dangi, AnilHuang, ChaoMurase, NorikoKimura, ShokoStolz, Donna B Wilson, Gregory CLentsch, Alex Gandhi, Chandrashekhar R, A novel mouse model of depletion of stellate cells clarifies their role in ischemia/reperfusion-and endotoxin-induced acute liver injury. Journal 
of hepatology, 2014. 60(2): p. 298-305.

3. Title T., S. Tripathi, and K.L. Hartshorn, Defensins and cathelicidins in lung immunity. Innate immunity, 2010. 16(3): p. 151-159.

4. Swain, PNayak, SKNanda, PKDash, S, Biological effects of bacterial lipopolysaccharide (endotoxin) in fish: a review. Fish \& shellfish immunology, 2008. 25(3): p. 191-201.

5. Hochstein H., The LAL test versus the rabbit pyrogen test for endotoxin detection: Update 87. Pharm. Technol, 1987. 11(6): p. 124-129.

6. Fingola, Fernando F Albertino, Sheila RG de MP Abrantes, Shirley Zamith, Helena PS., Proposed reduction of the in vivo pyrogen test by the in vitro LAL assay for the quality control of anticrotallic, antiscorpion, antirabies and antitetanus sera. Toxicology in Vitro, 2019. 59: p. 292-299.

7. Raetz, Christian R Ulevitch, RICHARD J Wright, SD Sibley, CAROL H Ding, Aihao Nathan, CARL F, Gram-negative endotoxin: an extraordinary lipid with profound effects on eukaryotic signal transduction. The FASEB Journal, 1991. 5(12): p. 2652-2660.

8. Brandenburg, Klaus Howe, Jorg Gutsman, Thomas Garidel, Patrick, The expression of endotoxin activity in the Limulus test as compared to cytokine production in immune cells. Current medicinal chemistry, 2009. 16(21): p. 2653-2660.

9. Carlin G. and E. Viitanen, In vitro pyrogenicity of diphtheria, tetanus and acellular pertussis components of a trivalent vaccine. Vaccine, 2005. 23(28): p. 3709-3715.

10. Rietschel, Ernst T Kirikae, Teruo Schade, F Ulrich Mamat, Uwe Schmidt, Gunter Loppnow, Harald Ulmer, Artur J Zähringer, U Seydel, UDPFSMBH Di Padova, F, Bacterial endotoxin: molecular relationships of structure to activity and function. The FASEB Journal, 1994. 8(2): p. 217-225.

11. Bowman, John P McCammon, Sharee A Brown, Mark V Nichols, David S McMeekin, Tom A, Diversity and association of psychrophilic bacteria in Antarctic sea ice. Applied and Environmental Microbiology, 1997. 63(8): p. 3068-3078.

12. Staley J. T. and J. J. Gosink, Poles apart: biodiversity and biogeography of sea ice bacteria. Annual Reviews in Microbiology, 1999. 53(1): p. 189-215.

13. Beveridge, T.J., Structures of gram-negative cell walls and their derived membrane vesicles. Journal of bacteriology, 1999. 181(16): p. 4725-4733.

14. Roberts, K. J., B12 The Pyrogen Test. Endotoxins: Pyrogens, LAL Testing and Depyrogenation, 2007: p. 261.

15. Brandenburg, Klaus Seydel, Ulrich Schromm, Andra B Loppnow, Harald Koch, Michel HJ Rietschel, Ernst Th, Conformation of lipid A, the endotoxic center of bacterial lipopolysaccharide. Journal of Endotoxin Research, 1996. 3(3): p. 173-178.

16. Cai, Tong Zhang, Yuan He, Qing Liu, Qian Pei, Yusheng Chen, Chen Gao, Hua, The Suitability of Pyrogen Test in vitro with Human Peripheral Blood Mononuclear Cell for Haemorrhagic Fever with Renal Syndrome Bivalent Vaccine. Journal of Applied Virology, 2017. 6(3): p. 19-25.

17. Sandle T., Pharmaceutical Microbiology: Essentials for Quality Assurance and Quality Control. 2015: Woodhead Publishing.

18. Hartung, Thomas Aaberge, Ingeborg Berthold, Susanne Carlin, Gunnar Charton, Emmanuelle Coecke, Sandra Fennrich, Stefan Fischer, Matthias Gommer, Martin Halder, Marlies, Novel pyrogen tests based on the 
human fever reaction. ATLANOTTINGHAM-, 2001. 29(2): p. 99-124.

19. Horn, D. L., S. M. Opal, and E. LoMastro, Antibiotics, cytokines, and endotoxin: a complex and evolving relationship in gramnegative sepsis. Scandinavian journal of infectious diseases. Supplementum, 1996. 101: p. 9-13.

20. Hanage, W. and J. Cohen, Stimulation of cytokine release and adhesion molecule expression by-products of Viridans streptococci. The Journal of infectious diseases, 2002. 185(3): p. 357-367.

21. Daneshian, Mardas Wendel, Albrecht Hartung, Thomas von Aulock, Sonja. High sensitivity pyrogen testing in water and dialysis solutions. Journal of immunological methods, 2008. 336(1): p. 64-70.

22. Dehus, O., T. Hartung, and C. Hermann, Endotoxin evaluation of eleven lipopolysaccharides by whole blood assay does not always correlate with Limulus amebocyte lysate assay. Journal of endotoxin research, 2006. 12(3): p. 171-180.

23. Greisman, S. E. and R. B. Hornick, Comparative pyrogenic reactivity of rabbit and man to bacterial endotoxin. Proceedings of the Society for Experimental Biology and Medicine, 1969. 131(4): p. 1154-1158.

24. Wolff, S.M., Biological effects of bacterial endotoxins in man. Journal of Infectious diseases, 1973. 128(Supplement_1): p. S259S264.

25. Raetz, C.R., Biochemistry of endotoxins. Annual review of biochemistry, 1990. 59(1): p. $129-170$.

26. Antonella Di Paolo, Katia Forti, Lucia Anzalone, Sara Corneli, Martina Pellegrini, Giulio Severi, Monica Cagiola, "First evaluation of endotoxins in veterinary autogenous vaccines produced in Italy by LAL assay",Biologicals, Volume 55, 2018, Pages 71-73.

27. Bolden, Jay S Warburton, Rob E Phelan, Robert Murphy, Marie Smith, Kelly R De Felippis, Michael R Chen, Dayue, Endotoxin recovery using limulus amebocyte lysate (LAL) assay. Biologicals, 2016. 44(5): p. 434440.

28. Su, W. and X. Ding, Methods of endotoxin detection. Journal of laboratory automation, 2015. 20(4): p. 354-364.

29. Sheraba, Norhan Saif Diab, Mohamed Reda Yassin, Aymen Samir Amin, Magdy Aly Zedan, Hamdallah Hafez., A Validation study of the Limulus Amebocyte Lysate Test as an end-product endotoxin test for Polyvalent Horse Snake Antivenom. PDA journal of pharmaceutical science and technology, 2019: p. pdajpst. 2018.009522.

30. Williams K. L., Endotoxins: pyrogens, LAL testing and depyrogenation. 2007: CRC Press.

31. Tanaka Shigenori Aketagawa, Jun Takahashi, Shoji Shibata, Yuko Tsumuraya, Yoichi Hashimoto, Yohichi., Inhibition of highmolecular-weight- $(1 \rightarrow 3)-\beta$-d-glucandependent activation of a Limulus coagulation factor $\mathrm{G}$ by laminarin oligosaccharides and curdlan degradation products. Carbohydrate research, 1993. 244(1): p. 115-127.

32. Sandle T., Assessing process hold times for microbiological risks: bioburden and endotoxin. 2012.

33. Valentini, Sara Santoro, Giovanna Baffetta, Federica Franceschi, Sara Paludi, Marilena Brandini, Elisa Gherardini, Leonardo Serruto, Davide Capecchi, Barbara., Monocyteactivation test to reliably measure the pyrogenic content of a vaccine: An in vitro pyrogen test to overcome in vivo limitations. 
Vaccine, 2019. 37(29): p. 3754-3760.

34. Nachum R. and R. N. Berzofsky, Chromogenic Limulus amoebocyte lysate assay for rapid detection of gram-negative bacteriuria. Journal of clinical microbiology, 1985. 21(5): p. 759-763.

35. Novitsky T. J. and P. F. Roslansky, Quantification of endotoxin inhibition in serum and plasma using a turbidimetric LAL assay. Progress in clinical and biological research, 1985. 189: p. 181-196.

36. Sakti, Setyawan P Lucklum, Ralf Hauptmann, Peter Bühling, Frank Ansorge, Siegfried, Disposable TSM-biosensor based on viscosity changes of the contacting medium. Biosensors and Bioelectronics, 2001. 16(9-12): p. 1101-1108.

37. Pfeiffer M. and A. Weiss, Removal of LALtest interfering low molecular weight substances by ultrafiltration. Progress in clinical and biological research, 1987. 231: p. 251.

38. Da Silva, Cristiane Caldeira Presgrave, Octavio Augusto França Hartung, Thomas de Moraes, Aurea Maria Lage Delgado, Isabella Fernandes., Applicability of the Monocyte Activation Test (MAT) for hyperimmune sera in the routine of the quality control laboratory: Comparison with the Rabbit Pyrogen Test. Toxicology in Vitro, 2016. 32: p. 70-75.

39. Jin, Yanan Jia, Juanjuan Li, Chan Xue, Jianqi Sun, Jiabei Wang, Kaiyuan Gan, YalingXu, Jing Shi, Yaqin Liang, Xing-Jie, LAL test and RPT for endotoxin detection of CPT11/DSPE-MPEG 2000 nanoformulation: what if traditional methods are not applicable? Asian Journal of Pharmaceutical Sciences, 2018.

40. Sushruta M. and K. Anubha, An overview of Limulus amoebocyte lysate (LAL) test.
International Research Journal of Pharmacy (IRJP), 2011. 2(4): p. 67-71.

41.Vipond, Caroline Findlay, Lucy Feavers, Ian Care, Rory, Limitations of the rabbit pyrogen test for assessing meningococcal OMV based vaccines. ALTEX-Alternatives to animal experimentation, 2016. 33(1): p. 47-53.

42. Fennrich, Stefan Hennig, Ulrike Toliashvili, Leila Schlensak, Christian Wendel, Hans Peter Stoppelkamp, Sandra, More than 70 years of pyrogen detection: Current state and future perspectives. Alternatives to laboratory animals, 2016. 44(3): p. 239-253.

43. Franco, Elvira Garcia-Recio, Verónica Jiménez, Pilar Garrosa, Manuel Girbés, Tomás Cordoba-Diaz, Manuel Cordoba-Diaz, Damián Endotoxins from a Pharmacopoeial Point of View. Toxins, 2018. 10(8): p. 331.

44. Caldeira, C Freitas, JC Gimenes, I Silva, SA Cabello, P Presgrave, OA, Normal temperature variation in New Zealand white rabbits during restraint for preliminary pyrogen test. Pharmeuropa bio \& scientific notes, 2014. 2014: p. 118-123.

45. Palma, Linda Rossetti, Francesca Dominici, Sabrina Buondelmonte, Costantina Rocchi, Marco BL Rizzardi, Gian P Vallanti, Giuliana Magnani, Mauro , Determination of interference during in vitro pyrogen detection: development and characterization of a cellbased assay. Assay and drug development technologies, 2017. 15(2): p. 64-76. 